\title{
Novas evidências científicas na assistência nutricional em portadores de lesão por
}

\section{pressão}

\author{
New scientific evidence in nutritional assistance for patients with pressure injuries \\ Nueva evidencia científica en la asistencia nutricional a pacientes con lesiones por presión
}

Helder Matheus Alves Fernandes ORCID: https://orcid.org/0000-0003-2068-9071

Faculdade Nova Esperança de Mossoró, Brasil E-mail: heldermatheus10@hotmail.com

Elane da Silva Barbosa

ORCID: https://orcid.org/0000-0002-2668-8064 Universidade Estadual do Ceará, Brasil

E-mail: elanesilvabarbosa@hotmail.com

Lorena Santiago de Souza

ORCID: https://orcid.org/0000-0002-7530-1671

Faculdade Nova Esperança de Mossoró, Brasil E-mail: lorenass010399@gmail.com

Maria Antônia Moraes de Sousa ORCID: https://orcid.org/0000-0002-1703-9281 Faculdade Nova Esperança de Mossoró, Brasil E-mail: mariaantoniamoraiss1999@gmail.com

Renato Gondim de Oliveira

ORCID: https://orcid.org/0000-0001-7894-2418 Universidade Potiguar, Brasil

E-mail: renatogondm@gmail.com

Moisés Iasley Lima Vasconcelos ORCID: https://orcid.org/0000-0002-8724-1063 Centro Universitário Estácio do Ceará, Brasil E-mail: moisesasley@gmail.com

Luisa Ariel Rodrigues Gomes Firmino ORCID: https://orcid.org/0000-0001-9424-2411

Centro Universitário Estácio do Ceará, Brasil E-mail: luisaarielgomes@gmail.com

Mariane de Oliveira Sandes

ORCID: https://orcid.org/0000-0002-3757-7423

Centro Universitário Estácio do Ceará, Brasil E-mail: mariane.sande@outlook.com

Jane Lane de Oliveira Sandes

ORCID: https://orcid.org/0000-0001-8758-3998 Centro Universitário Maurício de Nassau, Brasil E-mail: jane_sandes_@hotmail.com

Francisco Matheus Crisostomo Pinheiro

ORCID: https://orcid.org/0000-0001-9309-2968

Centro Universitário Estácio do Ceará, Brasil

E-mail: crisostomomatheuscp@gmail.com

\section{Resumo}

As lesões por pressão (LPP) são resultantes de uma pressão prolongada sobre a pele, mediada pela pouca movimentação, sendo necessária a implementação de ações preventivas e terapêuticas aos sujeitos. Objetiva-se, neste estudo, apresentar as principais evidências e recomendações clínicas na assistência nutricional em pacientes com lesão por pressão. Trata-se de uma Revisão Sistemática, nas bases de dados LILACS, SciELO, MEDLINE e ScienceDirect, a partir dos seguintes descritores: Nutrição, Nutricionista, Lesão por Pressão e Estado Nutricional, na qual foram utilizados os operadores booleanos "AND" e "OR", realizando as distintas combinações nas bases de dados. Compuseram o corpus desta investigação 15 artigos científicos, incluindo um suplemento e dois livros, publicados nos últimos cinco anos. Deste modo, a fim de apresentar de forma organizada as evidências científicas em torno das LPP, foram elaborados três quadros contendo a seleção dos artigos, as principais recomendações nutricionais no manejo da LPP e os principais nutrientes recomendados para auxiliar no processo de cicatrização. Identificou-se que o estado nutricional é fator decisivo para recuperação e progresso para as lesões, sendo indicada ferramentas de triagem nutricionais para detecção precoce para implementar o plano de ação terapêutica. Portanto, conclui-se que a 
integração da equipe interdisciplinar na prevenção e no tratamento das LPP significa potencializar a produção de um cuidado em saúde integral e, ao mesmo tempo, equânime, por valorizar o sujeito na sua complexidade e singularidade humana com foco na qualidade de vida, longevidade e segurança do paciente.

Palavras-chave: Nutrição; Lesão por pressão; Nutricionista; Estado nutricional.

\begin{abstract}
Pressure injuries (LPP) are the result of prolonged pressure on the skin, mediated by little movement, requiring the implementation of preventive and therapeutic actions to the subjects. The aim of this study is to present the main evidence and clinical recommendations in nutritional care for patients with pressure injuries. It is a Systematic Review, in LILACS, SciELO, MEDLINE and ScienceDirect databases, based on the following descriptors: Nutrition, Nutritionist, Pressure Injury and Nutritional Status, in which the Boolean operators "AND" and "OR", Making the different combinations in the databases. The corpus of this investigation comprised 15 scientific articles, including a supplement and two books, published in the last five years. In this way, in order to present scientific evidence around the LPP in an organized way, three tables were prepared containing the selection of the articles, the main nutritional recommendations in the management of the LPP and the main nutrients recommended to assist in the healing process. It was identified that the nutritional status is a decisive factor for recovery and progress for the lesions, with nutritional screening tools indicated for early detection to implement the therapeutic action plan. Therefore, it is concluded that the integration of the interdisciplinary team in the prevention and treatment of PPL means enhancing the production of comprehensive health care and, at the same time, equitable, by valuing the subject in its complexity and human uniqueness with a focus on quality of life, longevity and patient safety.
\end{abstract}

Keywords: Nutrition; Pressure injury; Nutritionist; Nutritional status.

\title{
Resumen
}

Las lesiones por presión (LPP) son el resultado de una presión prolongada sobre la piel, mediada por poco movimiento, requiriendo la implementación de acciones preventivas y terapéuticas a los sujetos. El objetivo de este estudio es presentar las principales evidencias y recomendaciones clínicas en el cuidado nutricional de pacientes con lesiones por presión. Se trata de una Revisión Sistemática, en las bases de datos LILACS, SciELO, MEDLINE y ScienceDirect, basada en los siguientes descriptores: Nutrición, Nutricionista, Lesión por presión y Estado nutricional, en la que los operadores booleanos "Y" y "O", realizando las diferentes combinaciones en las bases de datos. El corpus de esta investigación estuvo compuesto por 15 artículos científicos, incluido un suplemento y dos libros, publicados en los últimos cinco años. De esta forma, con el fin de presentar la evidencia científica en torno a la LPP de forma organizada, se elaboraron tres tablas que contienen la selección de los artículos, las principales recomendaciones nutricionales en el manejo de la LPP y los principales nutrientes recomendados para ayudar en el proceso de curación. . Se identificó que el estado nutricional es un factor decisivo para la recuperación y el avance de las lesiones, con herramientas de cribado nutricional indicadas para la detección temprana para implementar el plan de acción terapéutico. Por tanto, se concluye que la integración del equipo interdisciplinario en la prevención y tratamiento de la LPP significa potenciar la producción de una atención integral de salud y, al mismo tiempo, equitativa, al valorar el tema en su complejidad y singularidad humana con un enfoque de calidad de vida, longevidad y seguridad del paciente.

Palabras clave: Nutrición; Lesión por presión; Nutricionista; Estados nutricionales.

\section{Introdução}

Lesão por pressão (LPP) caracteriza-se como uma lesão na pele e/ou tecido subjacente, geralmente localiza-se sobre uma proeminência óssea, como resultado de pressão isolada, ou em combinação com cisalhamento. Logo, as lesões apresentam alta prevalência e incidência em pacientes hospitalizados, em diferentes níveis de complexidade do Sistema Único de Saúde (SUS), tanto em centros de cuidados primários, secundários, terciários ou em instituições especializadas para idosos ou pessoas com necessidades especiais. Assim, ocorre principalmente em pacientes restritos ao leito, visto que há um déficit de movimentação; diminuindo, portanto, consideravelmente a circulação sanguínea e, por conseguinte, comprometendo a qualidade de vida e capacidade funcional do sujeito (Mervis \& Phillips, 2019).

Desse modo, partindo da compreensão da fisiopatologia e do mecanismo das causas das LPP, as condições para o seu aparecimento ocorrem por um conjunto de fatores ambientais e fisiológicos propícios para o seu acontecimento, caracterizando-se como um quadro doloroso, associado a outras complicações como, por exemplo: desnutrição, presença de doenças crônicas, imobilidade no leito e uso de algumas drogas, como corticoides, entre outros. Logo, as LPP têm custo 
emocional e financeiro muito alto no que tange à suplementação nutricional e ao cuidado das feridas. Compactuando com essa vertente, os protocolos de segurança do paciente vêm justamente para amenizar ou neutralizar os mecanismos geradores das LPP (Meyer et al., 2019; AMB, 2011).

Nesse contexto, protocolos e programas de segurança do paciente nas instituiçõesde saúde devem ser planejados de forma multidisciplinar e interdisciplinar, visando o trabalho em equipe e a colaboração nas instituições, permitindo a elaboração de um plano de cuidado que consiga abranger os fatores de risco relacionados ao aparecimento dessas lesões. Além disso, o cuidado centrado no paciente a partir da articulação entre duas ou mais profissões, pode favorecer o diálogo, de forma que haja a promoção de uma abordagem integrada diferenciada (Araújo et al., 2017).

Para tanto, o tratamento/a assistência nutricional, na qual se insere o nutricionista, deverá envolver desde a reparação e amenização das LPP através dos suplementos nutricionais ou da alimentação do paciente, o controle das doenças associadas à essa enfermidade: Diabetes Mellitos (DM), Hipertensão Arterial Crônica (HAS), Dislipidemia, Doença Renal Crônica/ou Aguda e outras Doenças Crônicas Não Transmissíveis (DCNT), recuperação do estado nutricional e diminuição do catabolismo proteico que estão bastante presentes nesses pacientes, uma vez que impedem a cicatrização da ferida e evolução positiva do quadro clínico (Yamanaka, Okada \& Sanada, 2017; AMB, 2011).

A oferta da terapia nutricional individualizada é primordial para a evolução e melhora das LPP. Assim, a realização de ferramentas de triagem nutricionais para identificar os quadros de desnutrição ou risco nutricional são fundamentais para uma prevenção e um tratamento mais eficaz desse tipo de lesão, visto que o estado nutricional desnutrido ou subnutrido aumenta os riscos de desenvolvimento das lesões e retardo da cicatrização, caso a desnutrição já esteja instalada. Logo, é imprescindível que o paciente disponha de uma avaliação nutricional completa e individualizada, para ter uma ingestão suficiente de nutrientes, suplementação adequada e distribuição apropriada da pressão corporal (Van Anholt et al., 2010; Yamanaka, Okada, Sanada, 2017).

Nesse contexto, a Diretoria da Sociedade Brasileira de Nutrição Parenteral e Enteral (BRASPEN) teve a iniciativa de apresentar um Journal suplementar, lançado no segundo semestre de 2020 para a campanha "DIGA NÃO À LESÃO POR PRESSÃO" reunindo, de forma sintética, todas as evidências clínicas e científicas que se tem atualmente sobre assistência e manejo nutricional nos pacientes com LPP, visando estimular a padronização dos cuidados multiprofissionais de pacientes graves que apresentam a comorbidade. Além do mais, é de suma importância que a terapia nutricional seja parte integral do tratamento de pacientes graves, desnutridos ou em risco de desnutrição (BRASPEN, 2020).

Portanto, objetiva-se, neste estudo, apresentar as principais evidências e recomendações clínicas na assistência nutricional em pacientes com lesão por pressão.

\section{Metodologia}

Trata-se de revisão sistemática de natureza qualitativo, a qual se propõe a responder uma pergunta específica de forma objetiva, sendo a mesma reprodutível. Assim, este tipo de pesquisa visa reduzir o viés através do uso de métodos explícitos, sendo sua investigação bem definida, delineada, transparente e replicável, avaliando de forma crítica, ordenada e abrangente os achados dos estudos (Donato, Donato, 2019). Para tanto, conforme Pereira et al. (2018) a pesquisa qualitativa possibilita uma coleta de dados de forma direta em que o pesquisador é o principal instrumento para essa coleta, nessa visão, os dados coletados são de preferência descritivo, por isso foram elaborados os quadros ilustrativos descrevendo as principais alterações científicas contendo na área da LPP e nutrição.

Nesse sentido, foram definidos como critérios de inclusão: documentos na íntegra online, em português e inglês. Isso porque, ao se realizar a busca em bases de dados, deparou-se com carências em estudos nacionais, do tipo observacionais e 
experimentais, entre eles, citam-se: caso controle, transversais e ensaios clínicos randomizados, publicados em revistas indexadas, nos últimos cinco anos, discorrendo acerca das contribuições da assistência do nutricionista e as principais evidências científicas e/ou clínicas dos protocolos nutricionais no cuidado a lesões por pressão para o público restrito ao leito. Os critérios de exclusão, por sua vez, foram: estudos de revisões, relatos de experiências, com crianças e/ou adolescentes e aqueles que abordassem outros tipos de feridas, e que não estavam restritos ao leito ou hospitalizados.

Somando-se a isso, destaca-se que, em decorrência da escassez de artigos que tratem sobre essa temática, conforme os critérios previamente elencados acima, a título de complementação da bibliografia, foi utilizada uma cartilha (suplemento) constituída por orientações gerais da BRASPEN, para dar mais embasamento científico na produção, visto que os suplementos lançados pela BRASPEN constituem-se um dos principais documentos que reúne evidências que permitem uma melhor conduta terapêutica conforme as investigações mais recentes em relação à área da lesão por pressão. Além disso, foram selecionados mais dois livros específicos de nutrição para abordar as funções e as fontes alimentares dos imunonutrientes citadas pela BRASPEN.

A busca ocorreu em quatro bases de dados: Literatura Latino-Americana e do Caribe em Ciências da Saúde LILACS , Scientific Electronic Library Online - SciELO, Medical Literature Analysis and Retrieval System Online (MEDLINE) e ScienceDirect, utilizando-se os seguintes descritores, padronizados e disponíveis nos descritores em Ciências da Saúde - DeCS: "Nutrição", "Nutricionista", "Lesão por Pressão" e "Estado Nutricional” e Medical Subject Headings (MeSH): "Nutrition", "Nutritionist", "Pressure Injury" e "Nutritional Status", utilizando os operadores booleanos "AND” e "OR" e realizando as distintas combinações/cruzamentos nas bases de dados.

A escolha dessas bases de dados ocorreu pelo fato de terem maior visibilidade científica na área dos cuidados em saúde em lesões por pressões que abrangem a assistência nutricional e os protocolos do nutricionista em conduzir o paciente que vivenciam essa comorbidade.

O levantamento realizou-se de dezenove a cinco de março de 2021, sendo feita leitura prévia dos títulos e resumos dos estudos e, caso tivesse dúvida quanto à sua adequação, era realizada a leitura do artigo completo. Então, a amostra se constituiu de 12 artigos, além de um suplemento da BRASPEN sobre o assunto e dois livros específicos de nutrição, perfazendo, num total de 15 produções científicas.

\section{Resultados}

Conforme o mapeamento dos artigos, obtiveram-se, na LILACS, 65 artigos; na ScienceDirect, 144 artigos; MEDLINE 359 artigos; e por fim, na Scielo, 85 artigos, perfazendo um total de 653 artigos encontrados conforme os distintos cruzamentos entre os descritores. Para tanto, após análise, seleção, elegibilidade e leitura na íntegra dos artigos, foram selecionados 12 artigos elegíveis para compor o corpus da pesquisa, além disso, foram incluídos um suplemento da BRASPEN e dois livros específicos de nutrição, totalizando 15 documentos.

No que concerne ao ano de publicação, quatro documentos foram de 2016 e 2020; três no de 2017, dois no ano de 2018 e 2019. Além disso, o suplemento da BRASPEN foi publicado no de 2020. Sua inclusão foi necessária, pois possibilitou a compreensão e a definição dos objetivos, indicadores, meios e intervenções que o nutricionista pode estar à frente no tratamento da lesão por pressão conforme as últimas evidências que as BRASPEN reuniram.

O maior quantitativo de artigos no ano de 2016 pode ser explicado pelo fato de que, no mesmo ano, ocorreu a publicação do Procedimento Operacional Padrão na Prevenção de Úlcera por Pressão realizado pela Empresa Brasileira de Serviços Hospitalares (EBSERH) do Núcleo de Segurança do Paciente que versava sobre os novos procedimentos e protocolos na prevenção dessas lesões, e também ocorreu a mudança da terminologia de úlcera por pressão para LPP pela National Pressure Ulcer Advisory Panel (NPUAP), entidade norte-americana que conduz pesquisas nesta área. 
Research, Society and Development, v. 10, n. 3, e13310313058, 2021

(CC BY 4.0) | ISSN 2525-3409 | DOI: http://dx.doi.org/10.33448/rsd-v10i3.13058

A partir dos resultados do presente estudo, foi elaborado um quadro, o qual apresenta os artigos de acordo com o ano, bem como os autores, título, indexação periódica e objetivo dos estudos.

Quadro 1 - Estudos incluídos na revisão sistemática em relação ao ano de publicação, aos autores, ao título do artigo, origem de publicação e objetivo, Mossoró/RN, fev., 2021.

\begin{tabular}{|c|c|c|c|c|c|}
\hline$N^{\circ}$ & Ano & Autores & Título & Origem da Publicação & Objetivo \\
\hline 01 & 2016 & Ham et al., & $\begin{array}{l}\text { Pressure ulcer development in trauma } \\
\text { patients with suspected spinal injury; } \\
\text { the influence of risk factors present in } \\
\text { the emergency department. }\end{array}$ & $\begin{array}{l}\text { International Emergency } \\
\text { Nursing }\end{array}$ & $\begin{array}{l}\text { Explorar a influência dos fatores de risco presentes na admissão do Departamento de Emergência em } \\
\text { desenvolvimento de úlcera de pressão em pacientes com trauma com suspeita de lesão medular, } \\
\text { internados no hospital para avaliação e tratamento de lesões traumáticas agudas. }\end{array}$ \\
\hline 02 & 2016 & Cereda et al., & $\begin{array}{c}\text { Efficacy of a disease-specific } \\
\text { nutritional support for pressure ulcer } \\
\text { healing: a systematic review and meta- } \\
\text { analysis. }\end{array}$ & Journal Nutrient Health Aging. & $\begin{array}{l}\text { O objetivo desta revisão sistemática foi resumir as evidências sobre a eficácia de fórmula nutricional } \\
\text { rica em calorias e proteínas enriquecida com arginina, zinco e antioxidantes (específicos para } \\
\text { doenças suporte) em pacientes com úlceras de pressão (UPs). }\end{array}$ \\
\hline $\mathbf{0 3}$ & 2016 & Queiroz et al., & $\begin{array}{l}\text { Avaliação dos níveis séricos de } \\
\text { proteínas em pacientes com úlceras } \\
\text { por pressão. }\end{array}$ & Arquivos de Ciências da Saúde. & $\begin{array}{l}\text { Avaliar a interferência dos níveis séricos de proteína total, albumina e proteína } \mathrm{C} \text { reativa em } \\
\text { pacientes com úlceras por pressão. }\end{array}$ \\
\hline 04 & 2016 & Cereda et al., & $\begin{array}{l}\text { Cost-effectiveness of a disease- } \\
\text { specific oral nutritional support } \\
\text { for pressure ulcer healing. }\end{array}$ & Clinical Nutrition. & $\begin{array}{l}\text { Comparar as fórmulas nutricionais específicas para doenças, enriquecida com arginina, zinco e } \\
\text { antioxidantes, na melhora da a úlcera de pressão (UP) cicatrização em pacientes desnutridos. }\end{array}$ \\
\hline 05 & 2017 & Waitzberg, D.L. & $\begin{array}{l}\text { Nutrição Oral, Enteral e Parenteral na } \\
\text { Prática Clínica - } 1 \text { Vols. }\end{array}$ & Atheneu & $\begin{array}{l}\text { Tem por objetivo introduzir novos capítulos que levaram ao acréscimo de mais de } 20 \% \text { do seu } \\
\text { conteúdo. Além de apresentar as dietoterapias em diversos ciclos de vida, tanto nas condições de } \\
\text { terapia oral, enteral ou parenteral. }\end{array}$ \\
\hline 06 & 2017 & $\begin{array}{l}\text { Prado, Y.S.; } \\
\text { Tiengo, A.; } \\
\text { Bernardes, } \\
\text { A.C.B. }\end{array}$ & $\begin{array}{l}\text { A influência do estado nutricional no } \\
\text { desenvolvimento de lesões por pressão } \\
\text { em pacientes suplementados. }\end{array}$ & $\begin{array}{l}\text { Revista Brasileira de } \\
\text { Obesidade, Nutrição e } \\
\text { Emagrecimento. }\end{array}$ & Avaliar o estado nutricional e o desenvolvimento de lesões por pressão em pacientes suplementados. \\
\hline 07 & 2017 & $\begin{array}{l}\text { Oliveira, K.D.L.; } \\
\text { Hack, A.; Fortes, } \\
\text { R.C. }\end{array}$ & $\begin{array}{l}\text { Estado nutricional de idosos e } \\
\text { prevalência de lesão por } \\
\text { pressão na assistência domiciliar. }\end{array}$ & Revista enfermagem atual & $\begin{array}{l}\text { Avaliar o estado nutricional e determinar a prevalência de lesão por pressão (LPP) em idosos } \\
\text { assistidos por um serviço de atendimento domiciliar. }\end{array}$ \\
\hline $\mathbf{0 8}$ & 2018 & $\begin{array}{l}\text { Eglseer, D.; } \\
\text { Hodl, M.; } \\
\text { Lohrmann, C. }\end{array}$ & $\begin{array}{l}\text { Nutritional management of older } \\
\text { hospitalised patients with pressure } \\
\text { injuries. }\end{array}$ & Int Wound Journal & $\begin{array}{l}\text { Descrever as intervenções nutricionais conduzidas na Áustria pacientes hopitalizados, que tinham } 70 \\
\text { anos de idade ou mais e teve (risco de desenvolver) lesões por pressão. }\end{array}$ \\
\hline & & Souza, M.F.C.; & Risco de lesão por pressão em UTI: & & Realizar a adaptação transcultural da Escala de Valoración Actual del riesgo de desarrollar Úlceras \\
\hline
\end{tabular}


Research, Society and Development, v. 10, n. 3, e13310313058, 2021

(CC BY 4.0) | ISSN 2525-3409 | DOI: http://dx.doi.org/10.33448/rsd-v10i3.13058

\begin{tabular}{|c|c|c|c|c|c|}
\hline 09 & 2018 & $\begin{array}{l}\text { ZANEI, S.S.V.; } \\
\text { Whitaker, I.Y. }\end{array}$ & $\begin{array}{l}\text { adaptação transcultural e confi } \\
\text { abilidade da EVARUCI. }\end{array}$ & Acta Paul Enfermagem & $\begin{array}{l}\text { por presión en Cuidados Intensivos (EVARUCI) para a língua portuguesa do Brasil e analisar sua } \\
\text { confiabilidade em pacientes de Unidade de Terapia Intensiva (UTI). }\end{array}$ \\
\hline 10 & 2019 & Yap et al., & $\begin{array}{l}\text { Influence of Nutrition and } \\
\text { Nonnutrition Factors on Pressure } \\
\text { Injury Outcomes Among At-Risk } \\
\text { Asian Nursing Home Residents. }\end{array}$ & Advances in skin \& wound care & $\begin{array}{l}\text { Descrever as diferenças na nutrição geral, ingestão alimentar e não nutrição fatores de risco para IPs } \\
\text { entre residentes de NH asiáticos e não asiáticos. }\end{array}$ \\
\hline 11 & 2019 & Wong et al., & $\begin{array}{l}\text { Economic Evaluation of Nutrition } \\
\text { Support in the Prevention and } \\
\text { Treatment of Pressure Ulcers in Acute } \\
\text { and Chronic Care Settings: A } \\
\text { Systematic Review. }\end{array}$ & $\begin{array}{l}\text { Journal of Parenteral and } \\
\text { Enteral Nutrition }\end{array}$ & $\begin{array}{l}\text { Determinar o custo-efetividade e resultados clínicos do suporte nutricional na prevenção e tratamento } \\
\text { de UP. }\end{array}$ \\
\hline 12 & 2020 & $\begin{array}{l}\text { Cozzolino, } \\
\text { S.M.F. }\end{array}$ & $\begin{array}{l}\text { Biodisponibilidade de Nutrients }-6^{\circ} \\
\text { Edição. }\end{array}$ & Manole & $\begin{array}{l}\text { Tem por objetivo introduzir novos capítulos com o intuito de acompanhar alguns temas polêmicos, } \\
\text { como as questões ligadas ao glúten e a proteínas com potencial alergênico. Além de contribuir para } \\
\text { os fundamentos científicos no sentido de possibilitar ao leitor um posicionamento crítico quanto a } \\
\text { dietas restritivas, nem sempre necessárias. }\end{array}$ \\
\hline 13 & 2020 & BRASPEN & $\begin{array}{l}\text { Campanha Diga Não à Lesão por } \\
\text { Pressão. }\end{array}$ & $\begin{array}{l}\text { Brazilian Society of Parenteral } \\
\text { and Enteral Nutrition }\end{array}$ & $\begin{array}{l}\text { Difundir conhecimento, oportunizando suporte técnico ao cuidado do paciente e reduzir os índices de } \\
\text { LP. }\end{array}$ \\
\hline 14 & 2020 & Oliveira et al., & $\begin{array}{l}\text { Manejo nutricional de pacientes com } \\
\text { Lesão por Pressão em Terapia } \\
\text { Intensiva. }\end{array}$ & $\begin{array}{l}\text { Brazilian Journal of health } \\
\text { Review }\end{array}$ & $\begin{array}{l}\text { Analisar e comparar a dieta prescrita pelo o profissional de nutrição com as recomendações da } \\
\text { literatura, nos aspectos de oferta calórica e proteica. }\end{array}$ \\
\hline 15 & 2020 & Munoz et al., & $\begin{array}{l}\text { The Role of Nutrition for Pressure } \\
\text { Injury Prevention } \\
\text { and Healing: The } 2019 \text { International } \\
\text { Clinical Practice Guideline } \\
\text { Recommendations. }\end{array}$ & Clinical Management & Revisar as questões relacionadas à nutrição recomendações apresentadas na European Pressure 2019. \\
\hline
\end{tabular}

Fonte: Elaboração dos Autores (2021).

Para apresentar sistematicamente os resultados realizadas na presente investigação, foi elaborado um segundo quadro contendo as recomendações nutricionais conforme o grau

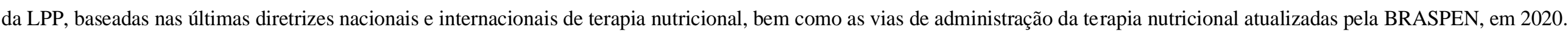




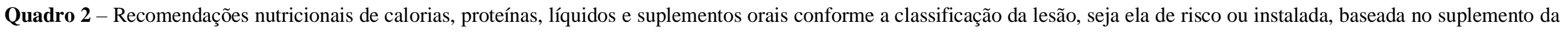
BRASPEN, Mossoró/RN, fev., 2021.

Nutrientes

Calorias no paciente estável

\section{Calorias no paciente crítico}

\section{Calorias no paciente obeso}

\section{Proteínas}

\section{Líquidos}

\section{Suplementos nutricional oral (SNO)}

\section{Risco de LPP}

LPP Instalada

Desnutridos ou em risco nutricional 30-
$35 \mathrm{kcal} / \mathrm{kg} / \mathrm{dia}$.

15 a $20 \mathrm{kcal} / \mathrm{kg} /$ dia do $1^{\circ}$ ao $3^{\circ} \mathrm{dia} ; 25$ a $30 \mathrm{kcal} /$ dia após o $4^{\circ}$ dia dos pacientes em recuperação.

11 a $14-\mathrm{kcal} / \mathrm{kg} / \mathrm{dia}$ de peso real (IMC entre $11-14 \mathrm{kcal} / \mathrm{kg} /$ dia do peso ideal (IMC entre $30-50 \mathrm{~kg} / \mathrm{m}^{2}$ ) e $22-25 \mathrm{kcal} / \mathrm{kg} / \mathrm{dia} \mathrm{do} \mathrm{peso}$ ideal, (IMC > $\left.30-50 \mathrm{~kg} / \mathrm{m}^{2}\right)$ e $22-25 \mathrm{kcal} / \mathrm{kg} / \mathrm{dia}$ do peso ideal, $\left(\mathrm{IMC}>50 \mathrm{~kg} / \mathrm{m}^{2}\right)$.

1,25 a $1,5 \mathrm{~g} / \mathrm{kg} / \mathrm{dia}$. Logo, se for pacientes renais, precisa avaliar a condição clínica.

$\mathbf{1 m l}$ de líquidos/kcal/dia.

Se baixa aceitação alimentar $(<60 \%$ das necessidades) precisa avaliar a necessidade de SNO hiper proteica no contexto da dieta ofertada.

Fonte: Elaboração dos Autores (2021).
1,5 a $2 \mathrm{~g} / \mathrm{kg} / \mathrm{dia}$. Logo, se for pacientes renais, precisa avaliar a condição clínica.

\section{$\mathbf{1 m l}$ de líquidos/kcal/dia.}

Introduzir SNP específico para cicatrização (contendo nutrientes específicos: zinco, arginina, carotenoides, vitaminas A,C e E).

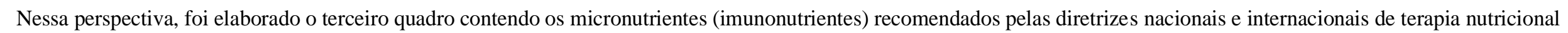

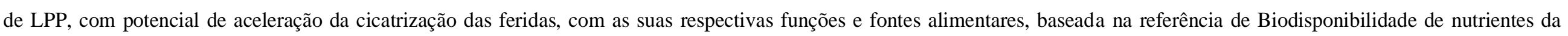
autora Silvia M. Franciscato Cozzolino e Nutrição Oral Enteral e Parenteral na Prática Clínica do autor Dan Linetzky Waitzberg. 
Quadro 3 - Categoria dos micronutrientes imunomodulador no processo de cicatrização das lesões com as respectivas funções e fontes alimentares, Mossoró/RN, fev., 2021.

\begin{tabular}{|c|c|c|}
\hline Nutrientes & Funções & Fonte \\
\hline Arginina & $\begin{array}{l}\text { Promove reparação tecidual por aumento da síntese de colágeno; apresenta ação imunoestimulante; promove } \\
\text { liberação da secreção de prolactina, insulina, GH e IGF; é percussora do óxido nítrico (Cozzolino, 2016; } \\
\text { Waitzberg, 2017). }\end{array}$ & Nozes e oleaginosas \\
\hline Zinco & $\begin{array}{l}\text { Participa de reações que envolve síntese ou degradação de metabólicos de carboidratos, lipídeos e proteínas; } \\
\text { atua como regulador do apetite; reduz o estresse oxidativo através da inibição de NADPH redutase, como } \\
\text { cofator da superóxido dismutase, além da melotioneína (Cozzolino, 2016; Waitzberg, 2017). }\end{array}$ & $\begin{array}{l}\text { Leite e derivados, ostras e mariscos, grãos e } \\
\text { cereais integrais, feijão seco e nozes. }\end{array}$ \\
\hline Carotenoide & $\begin{array}{l}\text { Função de pró-vitamina A; antioxidante e combate os radicais livres; ação enzimática e mecânica (Cozzolino, } \\
\text { 2016; Waitzberg, 2017). }\end{array}$ & $\begin{array}{l}\text { Alimentos com coloração do amarelo ao } \\
\text { vermelho nos frutos, ou seja, banana, } \\
\text { morango, tomate, cereja, etc. }\end{array}$ \\
\hline Vitamina A & $\begin{array}{l}\text { Sua ação é no ciclo visual; participando desde o crescimento, visão e integridade estrutural e/ou funcional do } \\
\text { tecido epitelial, reprodução e formação de dentes e ossos. Atua na síntese proteica e de membranas celulares, } \\
\text { além de proteção de barreira mucosa (Cozzolino, 2016; Waitzberg, 2017). }\end{array}$ & Fígado, leite, ovos, queijos e manteiga. \\
\hline Vitamina $\mathbf{C}$ & $\begin{array}{l}\text { Sua principal função é ser antioxidante; fomenta a produção e manutenção do colágeno; reduz a } \\
\text { susceptibilidade de infecções e participa no processo de cicatrização (Cozzolino, 2016; Waitzberg, 2017). }\end{array}$ & $\begin{array}{l}\text { Frutas e hortaliças frescas. Laranja, kiwi, } \\
\text { goiaba, acerola, caju, brócolos e couve. }\end{array}$ \\
\hline Vitamina $\mathbf{E}$ & $\begin{array}{l}\text { Sua principal função é ser antioxidante; atua na ativação de peróxidos, preserva as funções de comunicação } \\
\text { celular (Cozzolino, 2016; Waitzberg, 2017). }\end{array}$ & $\begin{array}{l}\text { Óleos vegetais (soja, milho), ovos e } \\
\text { oleaginosas. }\end{array}$ \\
\hline
\end{tabular}

Fonte: Elaboração dos Autores (2021). 


\section{Resultados}

As proteínas relacionam-se diretamente com o sistema imunológico e são integrantes dos tecidos corporais, além disso desempenham funções como marcadores diretos/indiretos de desnutrição, dentre as quais, podem ser mencionadas: albumina e pré-albumina, as principais, utilizadas para determinar o estado nutricional associado com demais marcadores antropométricos, bioquímicos, história alimentar e anamnese clínica em pacientes que são acometidos pelas consequências das LPP (Oliveira, 2017; Munoz et al., 2020).

Assim, entre os fatores nutricionais que interferem no risco para o desenvolvimento de LPPs, estão: inadequação da ingestão dietética, especialmente proteína; baixo índice de massa corporal (IMC); perda de peso, para o qual, deve-se atentar se foi voluntário ou involuntário. O involuntário caracteriza-se por risco nutricional em triagens e rastreamento nutricionais, além de valores baixos de referências das pregas cutâneas e baixos níveis séricos de albumina (Oliveira, 2017).

Desse modo, faz-se necessário que o nutricionista adeque a quantidade de nutrientes, levando em consideração à biodisponibilidade e ao processo fisiológico do trato gastrointestinal (TGI), o que significa se está totalmente funcional ou parcialmente, condição do paciente, método de preparo ou infusão da dieta, sendo que a diminuição de nutrientes resulta em menor resistência tecidual, alterações metabólicas e na diminuição transporte de oxigênio e aumento de edemas, fraqueza geral e indisposição (Queiroz et al., 2016; Yap et al., 2019).

Nesse sentido, o profissional deve garantir o suprimento das necessidades nutricionais para a manutenção do estado nutricional e evitar as complicações ocasionadas pelo desiquilíbrio do estado nutricional, seja por obesidade, seja por desnutrição, recomendado conforme o quadro 02 baseado na BRASPEN. Além disso, também há a recomendação de vitaminas e minerais conforme o Projeto Diretrizes de Terapia Nutricional e Dietary reference intakes (DRIS), visto que a intervenção deve contemplar um plano nutricional individualizado necessário para garantir a adequação de nutrientes e hidratação (BRASPEN, 2020).

Nesse pressuposto, o estado nutricional comprometido pela deficiência nutricional está diretamente associado com o desenvolvimento das LPP e impedimento de cicatrização mais pertinente (Cereda et al., 2017). Assim, torna-se necessário o uso de fórmulas especificas para determinada condição clínica, enriquecidas com arginina, zinco, carotenoide, vitamina A, C e E, como explicitado no quadro 03. Essas fórmulas como suplementos orais utilizadas, utilizado por pelo menos oito semanas, está correlacionada com a melhor cicatrização das LPP em comparação com fórmulas padrões. Somando-se a essa situação, o uso dessa fórmula nutricional oral específica (enriquecidas ou suplementadas) para doenças, não apenas resulta em uma melhor cicatrização das LPP, mas também reduz os custos do cuidado local da LPP Do ponto de vista do sistema de saúde local (Prado, Tiengo, Bernardes, 2017; CEREDA et al., 2016).

Os pacientes hospitalizados e/ou domiciliares que recebem uma dieta com maior aporte proteico (dieta hiperprotéica) detém menos probabilidade de desenvolver LPP, posto que a proteína tem papel imprescindível na cicatrização, entre elas, destacamse: colágeno, elastinas, fibronectinas e outros que contribuírem para remissão dessas lesões. Então, vale destacar que o melhor aporte proteico associado com os imunomoduladores eles conseguem potencializar a cicatrização. Também é pertinente ponderar que, quanto maior for o tempo de internação, maior será a susceptibilidade do quadro de desnutrição, agravamento das LPP, inflamação e evolução negativa dos pacientes e aumento de custos de tratamento (Oliveira et al., 2020; Oliveira, Haack, Fortes, 2017).

Nesse panorama, o uso de protocolos clínicos e/ou ferramentas de triagem nutricional são considerados como melhores métodos para integrar e sistematizar as boas práticas de cuidados ao paciente. Isso porque conseguem auxiliar a uniformizar as condutas institucionais e as incorporações desses protocolos, os quais vão desde as prescrições médicas, 
Research, Society and Development, v. 10, n. 3, e13310313058, 2021

(CC BY 4.0) | ISSN 2525-3409 | DOI: http://dx.doi.org/10.33448/rsd-v10i3.13058

dietéticas e de enfermagem e algoritmos, permitindo expectativa de resultados bem definidos na evolução do paciente (Munoz et al., 2020; BRASPEN, 2020). Nessa perspectiva, recomenda-se que seja realizada a análise nutricional em todos os pacientes internados após sua admissão hospitalar e, dependendo do sujeito, sugerem-se ferramentas de mini avaliação nutricional (MAN) para idosos, e adultos a Nutritional Risk Screening (NRS - 2002) ou Malnutrition Universal Screening Tool (MUST) (BRASPEN, 2020).

Nessa visão, a terapia nutricional adequada com a implantação de protocolos parece melhorar conforme algumas evidências, visto que o sucesso da implementação e execução de um bom protocolo e a qualidade da terapia nutricional realizada, bem como uma melhor adesão ao tratamento e conduta nutricional realizados pelo nutricionista, o que perpassa a integralidade, a individualidade e a personalização do plano terapêutico (BRASPEN, 2020).

Logo, uma das últimas evidências demonstrada nas diretrizes nutricionais atuais no manejo da LPP, precisam incluir uma das seguintes características: consumo de uma dieta balanceada, fornecimento de alimentos enriquecidos e/ou suplementos orais entre as refeições e considerações sobre o uso de suporte nutricional, ou seja, terapia enteral e/ou parenteral, ou ainda se a ingestão oral é inadequada, uma vez que os aconselhamentos dietéticos sem impor um aumento substancial na ingestão de energia ou proteína pode ser insuficiente para reduzir a ocorrências das LPP (Wong et al., 2018).

Nesse panorama, a presença de indicadores estruturais de qualidade promove um monitoradamente adequado e preciso na oferta de informações sobre a gestão do cuidado e características da população-alvo. Essas, por sua vez, são bastante utilizadas para a identificação de eventos adversos na atenção hospitalar, direcionar novos treinamentos à equipe assistencial, indicar novos parâmetros de monitoramento, sazonalidade de demandas ou até questões epidemiológicas (Eglseer, Hodl, Lohrmann, 2018; BRASPEN, 2020).

Dessa forma, os indicadores contribuem para baixa prevalência das LPP naqueles hospitais que conseguem adotar um sistema de monitoramento adequado, mas, nem todos os hospitais conseguem oferece uma estrutura e ferramentas de alta densidade tecnológica para os pacientes, comprometendo ainda mais no aumento de números de casos de LPP em unidades hospitalares (Souza, Zanei, Whitaker, 2018).

Pensando nisso, uma das formas de minimizar ou até mesmo evitar os custos dos hospitais, algumas condutas e alguns indicadores podem ser adotados pelos profissionais das unidades, os quais podem ser compreendidos como medidas preventivas para contribuir também na baixa prevalência dessas lesões. Assim, uma das estratégias denomina-se de "reposicionamento" e "mobilização precoce", também conhecida por "mudança de decúbito" nesses pacientes, paralelamente realizada com avaliação regular da pele, visto que deve ser intensificada para ajudar a detectar o risco de LPP em uma fase inicial, a fim de aplicar intervenções preventivas alternativas (Ham et al., 2016).

Pensando na ideia de medidas preventivas, um dos aspectos que também devem ser considerados refere-se à utilização de instrumento específico, de estabilidade e de fácil aplicação, visto que auxiliará na avaliação precoce da LPP e, consequentemente, impedirá a progressão das lesões, melhorando a qualidade de vida dos pacientes. Um desses instrumentos seria o uso da Escala de Valoración Actual del riesgo de desarrollar Úlceras por presión en Cuidados Intensivos - EVARUC que avalia o risco de LPP em pacientes adultos sob cuidados intensivos (Souza, Zanei, Whitaker, 2018).

A prevenção, a intervenção e o tratamento da LPP requerem uma assistência sistematizada no cuidado centrado no paciente. $\mathrm{O}$ trabalho em conjunto de uma forma interdisciplinar e interprofissional na prática colaborativa podem ofertar ao paciente uma avaliação que contemple o máximo possível das necessidades específicas conforme a personalização do plano terapêutico, e também, no que concerne ao aporte nutricional adequado, ao planejamento de um plano de cuidados direcionado, trazendo melhorias para a qualidade da assistência prestada pela equipe multidisciplinar do estabelecimento (Wong et al., 2018). 
Assim, o processo de trabalho entre os profissionais da unidade compreende a reformulação de protocolos adaptados conforme a individualidade de cada paciente, na medida em que as diretrizes internacionais nas suas últimas recomendações da gestão das LPP enfatizam a relação interdependente entre três categorias profissionais importantes no tratamento das lesões, a saber: nutrição, enfermagem e intervenções médicas (Wong et al., 2018; Padro, Tiengo, Bernardes, 2017).

\section{Considerações Finais}

As dietas enriquecidas com nutrientes específicos no decorrer do processo de cicatrização estão indicadas em pacientes com LPP já instalada, porém, deve-se avaliar individualmente a condição pela equipe multidisciplinar de terapia nutricional (EMTN) e a necessidade de utilização. Logo, o estado nutricional é fator decisivo para recuperação e progresso, uma vez que o risco nutricional para todos os pacientes são fatores de risco para LPP, sendo indicada a realização da triagem nutricional em até $48 \mathrm{~h}$ após admissão hospitalar para todos os pacientes internados e dependendo do sujeito. Assim, sugere-se MAN para idosos, e adultos a NRS- 2002 ou MUST.

As novas recomendações no aporte calórico, proteico, líquidos, suplementos orais e protocolos nutricionais juntamente com seus indicadores de qualidades trouxeram a reformulação de condutas personalizadas nos cuidados a pacientes com LPP, visto que as evidências clínicas devem nortear a prática do nutricionista, adaptando-se às intervenções nutricionais conforme a realidade de cada serviço e paciente, levando em consideração clareza, concisão, formato e o fácil manuseio dietético no paciente.

Articular, portanto, o trabalho da equipe interdisciplinar na prevenção e no tratamento das LPP, significa potencializar a produção de um cuidado em saúde integral e, ao mesmo tempo, equânime, por valorizar o sujeito na sua complexidade e singularidade humana. Constata-se, assim, que a parceria entre enfermeiro, nutricionista e equipe médica de acordo com as recomendações das diretrizes internacionais de terapia nutricional precisam ser exploradas, o que pode ser evidenciado pelo déficit de produções que tratem dessa articulação, ou melhor, da exploração dessa conduta interdisciplinar, ou até mesmo interprofissional.

Por fim, as últimas diretrizes baseadas em evidências afirmam claramente que as intervenções nutricionais, isto é, triagem nutricional, encaminhamento a um nutricionista, lanches/alimentos enriquecidos com energia/proteína ou ONS na prática clínica, são explorados apenas em uma minoria dos pacientes, e que, não estão sendo adotadas as recomendações das evidências. Por isso, estudos futuros precisam ser realizados, com intervenções padronizadas e maior quantitativo de participantes a longo prazo, principalmente, intervenções focadas na realidade dos sistemas de saúde do Brasil, dado que possibilitam a criação de um modelo de intervenção nutricional baseado em evidências para melhorar o manejo e protocolo da terapia nutricional conforme a realidade local dos sujeitos que apresentam as lesões.

\section{Referências}

Araújo, T. A. M et al (2017). Multiprofissionalidade e interprofissionalidade em uma residência hospitalar: o olhar de residentes e preceptores. Interface. Botucatu. 21 (62), 601-13.

Cereda, E., et al (2017). Efficacy of disease-specific nutritional support for pressure ulcer healing: a systematic review and meta-analysis. J Nutr Health Aging. Itália. 1 (1), 1-6.

Cereda, E et al (2016). Cost-effectiveness of a disease-specific oral nutritional support for pressure ulcer healing. Clinical Nutrition. Itália. 1 (1), 1-7. Disponível em:

Cozzolino, S.M.F (2016). Biodisponibilidade de nutrientes (5a ed.), Manole.

Campanha Diga Não à Lesão por Pressão (2020). Sociedade Brasileira de Nutrição Parenteral e Enteral (BRASPEN). $1^{\circ}$ Suplemento. p.1-26. https://66b28c71-9a36-4ddb-9739 12f146d519be.usrfiles.com/ugd/66b28c_763bfa2916bc4dbbabef747b3c43de9b.pdf. 
Research, Society and Development, v. 10, n. 3, e13310313058, 2021

(CC BY 4.0) | ISSN 2525-3409 | DOI: http://dx.doi.org/10.33448/rsd-v10i3.13058

Donato, H e Donato, M. (2019). Etapas na Condução de uma Revisão Sistemática. Acta Med Port. Portugal, 32 (3), $227-235$.

Eglseer, D e Hodl, M (2018). Lohrmann, C. Nutritional management o folder hospitalised patients with pressure injuries. Wiley Online Library. Áustria. 1 (1), $1-6$.

Ham, H.W et al (2016). Pressure ulcer development in trauma patients with suspected spinal injury, the influence of risk factores presente in the emergency departamento. International Emergency Nursing. Utrecht. 1 (1), 1-7.

Munoz, N. et al (2020). The role of nutrition for pressure injury prevention and healing: the 2019 international clinical practice guideline recommendations. Advances in Skin e Wound Care. United State. 33 (3), 123-136.

Mervis, J. S \& Phillips, T. J (2019). Pressure ulcers: Prevention and management. Journal of the American Academy of Dermatology, United State. 81 (4), 893-902.

Meyer, D et al (2019). Knowledge of nurses and nursing assistants about pressure ulcer prevention: A survey in 16 Belgian hospitals using the PUKAT 2.0 tool. Journal of Tissue Viability, United State. 2 (28), 59-69.

Oliveira, K. D. L., Haack, A \& Fortes, R. C (2017). Estado nutricional de idosos e prevalência de lesão por pressão na assistência domiciliar. Revista Enfermagem Atual. Rio de Janeiro. 1 (1), 1-6.

Oliveira, D. R et al (2020). Manejo nutricional de pacientes com lesão por pressão em terapia intensiva. brazilian journal of health review. Curitiba. 3 (3), 6592-6602.

Prado, Y. S \& Tiengo, A (2017). Bernardes, A. C. B. A influência do estado nutricional no desenvolvimento de lesões por pressão em pacientes suplementados. Revista Brasileira de Obesidade, Nutrição e Emagrecimento. 11 (68), 699-709.

Pereira, A. S. et al. (2018). Metodologia da pesquisa científica. UFSM. https://repositorio.ufsm.br/bitstream/handle/1/15824/Lic_Computacao_MetodologiaPesquisa-Cientifica.pdf?sequence $=1$.

Queiroz, V. B. B et al (2016). Avaliação dos níveis séricos de proteínas em pacientes com úlceras por pressão. Arquivos de Ciências da Saúde. São José do Rio Preto. 23 (1), 89-92.

Rodríguez-Núñez, C et al (2019). Nursing records, prevention measures and incidence of pressure ulcers in an intensive care unit. Enfermería Intensiva, Canadá. 30 (3), 135-143.

Souza, M. F. C., Zanei, S. S. V., \& Whitaker, I. Y. (2018). Risco de lesão por pressão em UTI: adaptação transcultural e confiabilidade da EVARUCI. Acta Paulista de Enfermagem. São Paulo. 31 (2), 201-208.

Terapia Nutricional para Portadores de Úlceras por Pressão (2011). Associação Médica Brasileira e Conselho Federal (AMB). p.1-10. al.https://diretrizes.amb.org.br/_BibliotecaAntiga/terapia_nutricional_para_pacientes_portadores_de_ulceras_por_pressao.pdf.

Van Anholt, R. D. et al (2010). Specific nutritional support accelerates pressure ulcer healing and reduces wound care intensity in non-malnourished patients. Nutrition, Paris. 26 (9), 867-872.

Waitzberg, DL (2017). Nutrição oral, enteral e parenteral na prática clínica. Atheneu.

Wong, A. et al (2018). Economic evaluation of nutrition support in the prevention and treatment of pressure ulcers in acute and chronic care settings: a systematic review. Journal of Parenteral and Enteral Nutrition. Singapura. 1 (1), 1-24.

Yamanaka, H, Okada, S., \& Sanada, H. A. (2017). Multicenter, randomized, controlled study of the use of nutritional supplements containing collagen peptides to facilitate the healing of pressure ulcers. Journal of Nutrition \& Intermediary Metabolism, Europen. 8 (1), $51-59$.

Yap, T. L., et al (2019). Influence of nutrition and nonnutrition factors on pressure injury outcomes among at-risk asian nursing home residentes. Advances in Skin e Wound Care. Texas. 32 (10), 463-469. 